\title{
4.33 Pulmonary Vein Isolation for Atrial Fibrillation and
} Baroreflex-Mediated Heart Control

K. Styczkiewicz, 1 G. Spadacini,2 M. Tritto,2 P. Moretti, 2 G. Perego,3 M. Facchini, 3

G. Bilo,4 P. Castiglioni,5 M. Di Rienzo,5 K. Kawecka-Jaszcz,1 J.A. Salerno-Uriate,6

G. Parati4

(1) I Dipt. di Cardiologia e Ipertensione, Jagiellonian Univ, Cracovia, Po Poland;

(2) Dipartimento di Cardiologia Ist Mater Domini, UNiv dell'Insubria, Castellanza,

It Italy; (3) Istituto Auxologico Italiano, Mi Milano-Italy; (4) Univ.Milano-Bicocca,

Istituto Auxologico Italiano, Milan, It Italy; (5) Centro di Bioingegneria,

Fondazione Don Gnocchi, Milan, It Italy; (6) Ist Clinico Mater Domini e

Dipartimento Scienze Cardiovascolari, Ospedale Circolo e Fondaz Macchi,

Varese, It Italy

Introduction: The data concerning the effects of ablation procedures for treatment of atrial fibrillation (AF) on changes in autonomic cardiac control are suffering from inconsistency. Little is known on possible modulation of sensitivity of cardiac baroreflex (BRS). Aim of our study was to assess the time course of changes in BRS after ablation, and their possible relationship with AF recurrence.

Methods: In 20 patients (age:60.4 \pm 5.3 ) undergoing pulmonary vein isolation (PVI) for AF, beat-tobeat blood pressure and ECG monitoring was performed for $10 \mathrm{~min}$ at controlled breathing rate $(0.35$ $\mathrm{Hz}$ ) 1-2 days before, 1 month and 6 months after PVI. Baroreflex function was assessed by frequency domain BRS [Alpha coefficient and the modulus of PI/SBP transfer function $(\mathrm{H})$ at low (LF, 0.04-0.14 $\mathrm{Hz}$ ) and high frequency (HF, 0.15-0.50 Hz)] and by the slope of UP (Seq+/+) and DOWN (Seq-/-) PI/ SBP sequences. The degree of atria denervation after ablation was quantified as DegD = BRS preablation / BRS 1 month post-ablation.

Results: During 6 month follow-up after PVI AF recurrence was observed in 6 patients. Patients with and without $\mathrm{AF}$ recurrence did not display differences in clinical and echocardiographic measures. When separately considered, patients without AF recurrence, compared to baseline, 1 month after PVI displayed a significant BRS reduction (alphaLF $7.6 \pm 4.6$ vs $3.8 \pm 2.3 \mathrm{~ms} / \mathrm{mmHg}, \mathrm{p}=0.02$; alphaHF $11.0 \pm 4.4$ vs $6.5 \pm 1.3 \mathrm{~ms} / \mathrm{mmHg}, \mathrm{p}=0.02 ; \mathrm{H}-\mathrm{LF} 6.0 \pm 3.7 \mathrm{vs} 3.0 \pm 1.9 \mathrm{~ms} / \mathrm{mmHg}, \mathrm{p}=0.02 ; \mathrm{H}-\mathrm{HF} 8.8 \pm 3.4 \mathrm{vs}$ $5.1 \pm 1.0 \mathrm{~ms} / \mathrm{mmHg}, \mathrm{p}=0.02 ; \mathrm{Seq}+/+12.1 \pm 5.6$ vs $6.8 \pm 1.6 \mathrm{~ms} / \mathrm{mmHg}, \mathrm{p}=0.04 ; \mathrm{Seq}-/-11.1 \pm 4.3$ vs $6.1 \pm 2.2 \mathrm{~ms} / \mathrm{mmHg}, \mathrm{p}=0.02)$. BRS at 6 months after PVI returned to baseline values. Patients with AF recurrence were characterized by a different pattern with no significant changes in BRS after PVI. There was no correlation between DegD and the number of ablated PVs, the procedure duration and fluoroscopy time. In multivariate analysis DegD (for BRS calculated at LF) remained an independent predictor of AF recurrence over a 6 month follow-up( $\mathrm{p}=0.01$ )

Conclusions: The clinical success of PVI in preventing AF recurrence over 6 months seems to be related to the degree of transient parasympathetic atrial denervation reflected by BRS reduction 1 month after the procedure. This observation suggests the possible use of BRS as an index of PVI efficacy in patients with AF. The implications of these findings deserve a further evaluation in longitudinal studies in a larger group of patients. 